\title{
Lane Characterization Under Challenging Scenarios For Autonomous Driving System
}

\author{
${ }^{1}$ Varsha Singal, ${ }^{2}$ Susmitha Mohan and ${ }^{3}$ Upendra Suddamalla \\ 1, 2, 3 Embedded Innovation Lab, EIS, Tata Consultancy Services, Bangalore, India; \\ varsha.singal@tcs.com; susmitha.mohan@tcs.com; upendra.suddamalla@tcs.com
}

\begin{abstract}
Advanced Driver Assistance systems (ADAS) have seen increasing popularity due to their importance in automotive driver safety and autonomous driving. These systems analyze data from several sensors mounted on the vehicle to detect lanes, obstacles and traffic conditions to ensure safe driving. Lane markings on road with different color and structure provide information on safe drive zone and other traffic restrictions on road. Typical ADAS solutions depend on vision based sensors for lane detection. Here we propose an efficient algorithm for detecting type and color of the lane marks as this information plays critical role in taking the decision for safety features such as lane change and lane keep assist. Our algorithm is pluggable to any state-of-art lane detection algorithm and provides lane type and color for straight, curvy roads. The proposed method is tested on various challenging scenarios and results are promising.
\end{abstract}

Keywords: Lane Detection, Lane Color, Lane Structure, Relative Color, Zero Crossing, Driver Assistance, Autonomous driving

\section{Introduction}

Detecting lane markings is one of the primary requirement of ADAS and autonomous driving solutions. Typically this is performed using vision based sensors mounted on the vehicle. Along with the lane position information, it is important to identify the type and color of the lane marks. The lane type includes solid and dashed with varying gaps between dashes and lane colors vary between white and yellow in different geographies [1]. Accurate detection of these lane characteristics plays very important role in the driver assistance features such as Lane Keep Assistance (LKA), Lane Departure Warning (LDW) etc. Varying illumination conditions and varying sensor color temperature cause challenges to vision based algorithms for lane detection and characterization. Sample scenarios are depicted in Fig. 1.
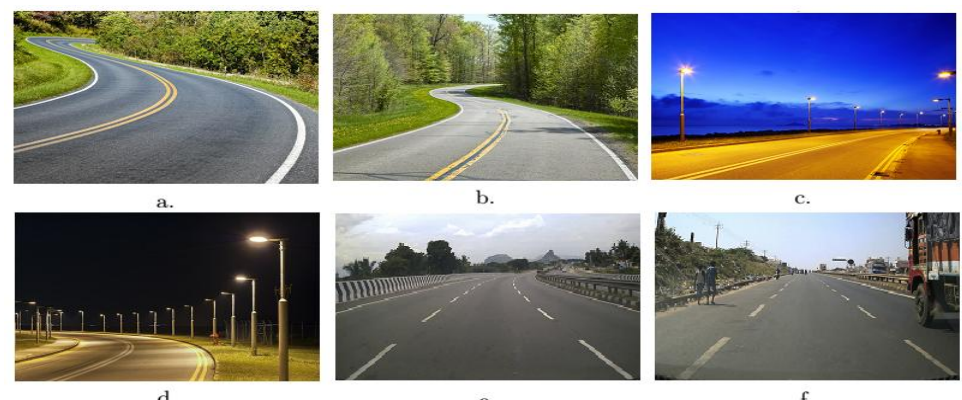

Figure 1 Scenarios indicating varying driving environment for lane type and lane color 
Several lane detection algorithms are proposed based on low level features such as edge and curve fitting [2], shape and structure of lane [3], histogram based [4] and lane models [2]. Performance of the lane detection algorithms vary based on the image quality and illumination levels. After detecting lane mark position, the images need to be processed for lane characteristics. The lane marking characteristics indicate different traffic restrictions in different geographies. Below Table I indicates widely referred meaning of these characteristics.

Table 1 Significance of lane type and lane color

\begin{tabular}{|c|c|c|}
\hline Lane Type & Lane Color & Significance \\
\hline Solid & White & Lane switch not allowed \\
\hline Dashed & White & Lane switch allowed \\
\hline Solid & Yellow & Never switch the lane \\
\hline Dashed & Yellow & Lane switch allowed if safe \\
\hline
\end{tabular}

Multiple state-of-art techniques have been proposed for lane type and lane color detection. Classification based method proposed by Rodrguez et al. [5] uses spatial descriptors and frequency domain analysis. Mauricio et al. [6] have proposed a binary classifier to distinguish different types of lane marking. Stefan et al. [7] have proposed a classification method for lane type detection. IPM based method proposed by Juan et al. [4] explains bird eye view approach on the captured images which is used for the lane type detection by applying Fourier analysis. Suchitra et al. [8] have proposed a method which uses spatial and temporal continuity analyses for lane type detection. For lane color, segmentation in HSV color space is proposed by Boggavarapu et al. [9] have proposed a method for determining the color of road marking using Support Vector Machines. Trung et al. [10] used HSI color space model for lane detection marking. Hyun et al. [11] proposed neural network based lane color detection method. Lee et al. [12] proposed a method to detect lane color using support vector machine. However, the classifier based methods for lane type [5], [6], [7] are highly data dependent and computationally intensive and histogram methods [4] face issues in curvy road scenarios. Color segmentation methods for lane color [9], [10] suffer during illumination variations and image sensor settings which are very common in autonomous driving environment. So it is very important for the algorithm to identify lane characteristics accurately under challenging scenarios such as varying illumination condition, sensor color temperature.

In the proposed method, we focus on lane characterization and for this we take output of any stateof-art lane detection algorithm as input. Most of the lane detection algorithms perform well in near regions due to good visibility and less noise. Taking this as an advantage, we consider the lane detection details in nearest zone over subsequent frames and perform statistical analysis for lane characterization. Section 2 describes the method of lane detection using mask based approach which produces the input for the proposed algorithm. The approach for lane characterization is described in Section 3, which defines the method to detect the lane type and the lane color. Details of the experiments and results are discussed in Section 4. Finally, the conclusions are presented in Section 5.

\section{Lane Detection}

Reliable detection of lane marks in a given scene is a primary requirement for the proposed lane characterization algorithm. This algorithm can use output from any state-of-art lane detection algorithm, provided the algorithm gives accurate position and start, end points of the lane in given scene. In the current work, we have used the mask based approach for lane detection as explained in [3]. This algorithm uses edge and lane mask as the key features and is capable of detecting lanes in both straight road and curvy road as shown in Fig.2. The algorithm detects inner boundary of the lane 
Varsha Singal, Susmitha Mohan and Upendra Suddamalla; Lane Characterization Under Challenging Scenarios For Autonomous Driving System. Advances in Image and Video Processing, Volume 4 No 6, December (2016); pp: $18-26$

marks in both the cases of single lane marking or double lane marking. The lane detection results of the algorithm for different scenarios is as shown in Fig.3. The interpolation of output between dashes during post processing is suppressed to get the actual lane points in case of dashed lane. From the algorithm results, the start and end points of each lane marking are obtained, which is then passed as input for lane characterization.

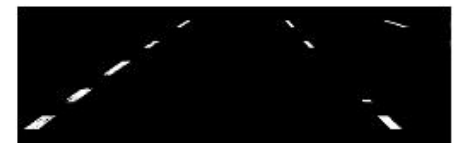

a. Mask Image

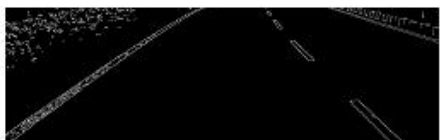

b. Edge image

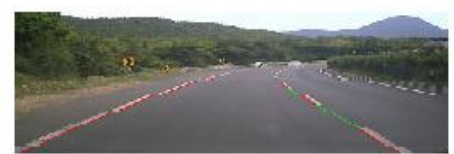

c. Lane output

Figure 2 Mask based lane detection

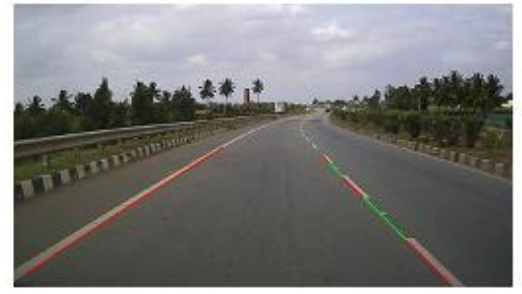

a.

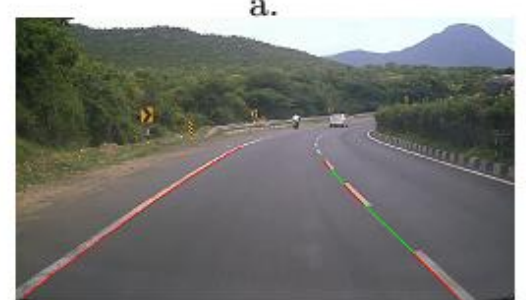

c.

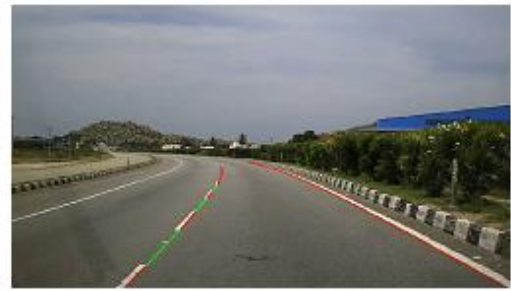

b.

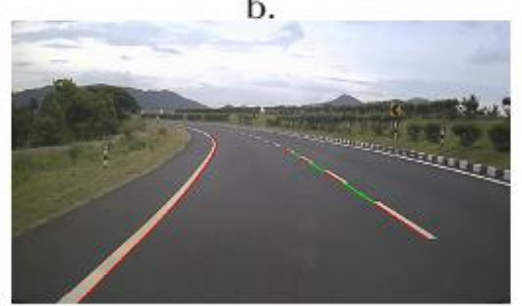

d.

Figure 3 Lane detection output for different scenarios

\section{Lane Characterization}

The lane information obtained from lane detection algorithm is used for identifying the type and color of the lane mark. Here we use a pre-defined near zone in front of the vehicle for example, up to 10 meters, and determine the characteristics of the lane marks detected in the selected zone. The near zone is considered for the reason that this zone has good contrast and visibility. The lane detection algorithm output is processed to shortlist the lane segments present in the selected zone. Start and end points of these shortlisted lane segments in the selected zone are accumulated for a sequence of $\mathrm{N}$ frames which will be used for lane type and lane color determination. Figure. 4 shows the image zone selected for determining type and color of the lane marking.

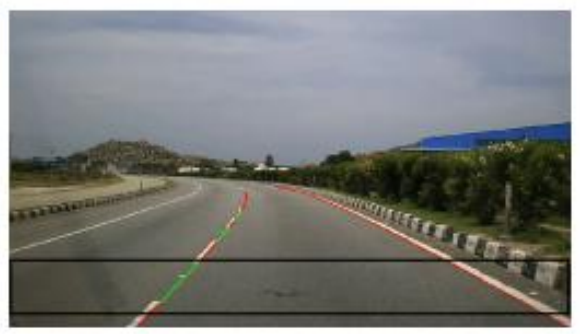

Figure 4 Zone selection 


\subsection{Lane Type}

Continuous pattern of solid lane and periodic nature of dashed lane are useful information to detect the type of lane. Here we propose a method to find the continuity and periodicity of lane in a predefined zone of the frame. Start points of the shortlisted lane marks in the selected zone are stored into an array. This data is accumulated for both left and right lane separately for ' $N$ ' number of frames. The distance of start point (lane top y) from beginning of zone (zone top y) is then calculated as the StartPointDistance. Variation of this distance across ' $N$ ' frames is analyzed for finding the lane type. Fig. 5 shows the plot of start point distance in solid and dashed lane scenarios. The plots clearly indicate the periodic pattern in case of dashed lane mark and very little variations for solid lane mark. Small variations in the solid lane scenario are due to image quality, digitization and approximations introduced by the lane detection algorithm. To handle these minute variations, we have used StartDistOffset.

This

offset is considered as ZeroReference shown in Fig. 5 and the distances below this offset are considered as zero. Dashed lanes exhibit a periodic pattern and the periodicity varies based on speed of the vehicle and then gap between the dashes. In contrast to the auto regression and frequency domain based techniques, which are computationally complex [4], here we propose a statistical analysis based technique. StartPointDistance for the previous ' $\mathrm{N}$ ' frames are analysed to determine the zero crossings with respect to ZeroReference. The mean and variance of zero crossing distances is validated to check if the lane marks are dashed.

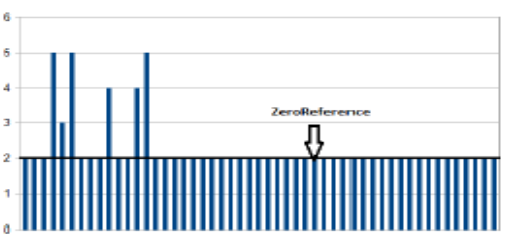

a. Start Point distance for solid

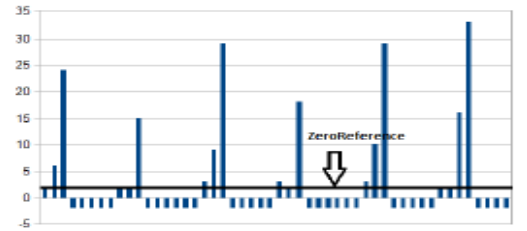

d. Start Point Distance for dashed

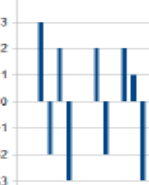

b. Start point distance difference for solid

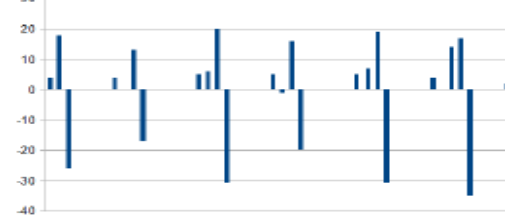

e. Start point distance difference for dashed

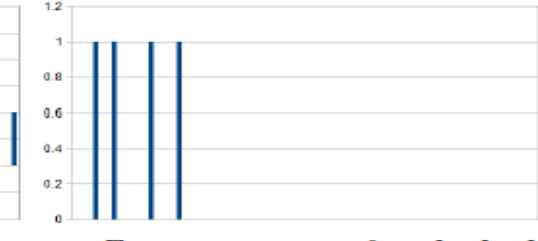

c. Zero-crossover for dashed

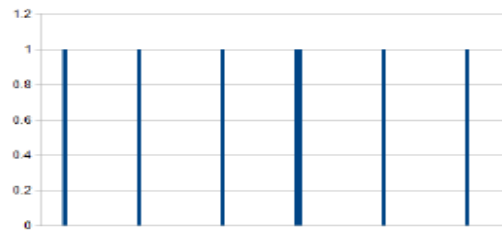

f. Zero crossover for dashed

Figure 5 Plot indicating start point distance, start point distance difference and zero-crossover

The formulae used for the calculation of ZeroCrossover, Mean and Variance are given in Eq. 1, 3, 4.

$$
\begin{aligned}
& \text { if }((\text { StartPointDist } / i+1 / \text { - StartPointDist } / i])<0) \\
& \text { where } i=1 \text { to } N-1 \\
& \text { ZeroCrossover }[j]=i \text {; } \\
& \text { where } j \text { varies from } 1 \text { to } M \text { (number of zero crossover } \\
& \text { points) } \\
& \text { ZeroCrossoverDist }[k]=\text { ZeroCrossover }[i+1] \\
& - \text { ZeroCrossover }[i] \text {; } \\
& \text { Mean }=\frac{1}{K} \sum_{k=1}^{K}(\text { ZeroCrossoverDist }[k]) \\
& \text { Variance }=\frac{1}{K-1} \sum_{k=1}^{K}(\text { ZeroCrossoverDist }[k]-\text { Mean })^{2}
\end{aligned}
$$

where $K$ varies from 1 to $M-1$

Pseudo code for lane type is as follows 


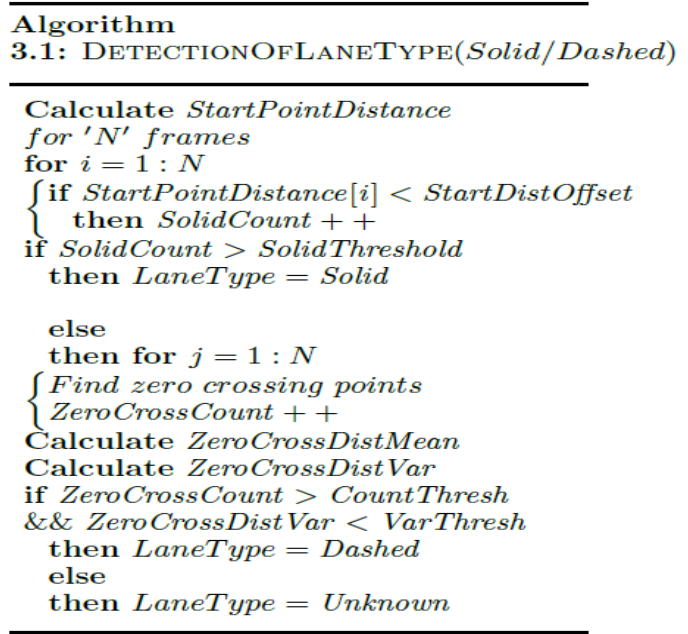

Any undefined pattern due to lane marking quality or limitations of lane detections algorithm is classified as UNKNOWN.

The lane type output under different scenarios are shown in Figure 6
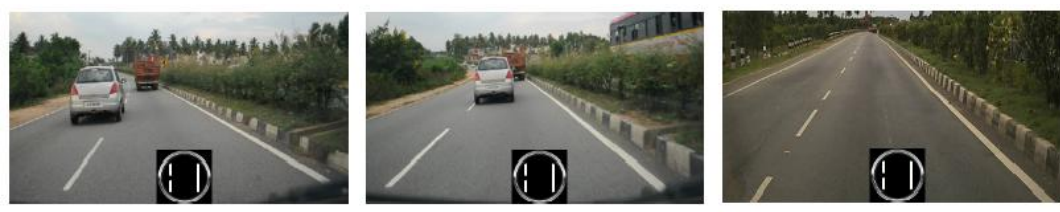

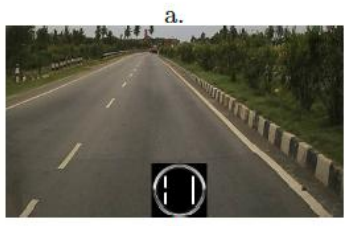

d.

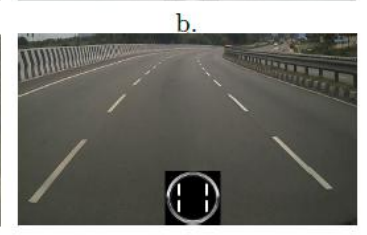

e.

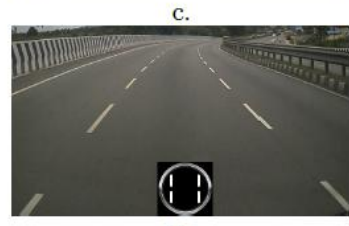

f.

Figure 6 Lane type Output

\subsection{Lane Color}

Different colored lane markings are used to indicate traffic restrictions in a given region. White and yellow colored lane markings are used widely in most of the geographies. Various color spaces are defined to determine the color and several methods are proposed for the same [9]. But real time ADAS systems include varying illumination conditions, shadows, environment conditions and image sensor settings. These cause very challenging scenarios for basic color detection techniques. Here we analyzed the usage of HSV color space for determining the color of lane markings which can be white or yellow. With reference to the earlier work on determining yellow color [9], we have defined a customized range for yellow lane marks. The ranges are as shown in Table 2.

Table 2 HSV range for yellow and white color

\begin{tabular}{|l|c|c|c|}
\hline & Hue & Saturation & Value \\
\hline Yellow & 20 to 60 & 140 to 255 & 100 to 255 \\
\hline White & 0 to 255 & 0 to 80 & 100 to 255 \\
\hline
\end{tabular}

These ranges are found to be working well under proper lighting conditions, but failed in segregating white and yellow color in night condition with halogen lights or different color temperature settings of imaging sensor. Some of the conditions where this method is failing is shown in Fig.3. Here the basic color detection algorithms classified white lane as yellow lane because of the matching ranges of HSV. 
As these scenarios are common in ADAS systems, the algorithm should be capable of handling these conditions.

Here we propose a novel method based on relative color and saturation of the lane marking with respect to its neighbourhood road region. The yellowness of the lane marking is validated based on the relative yellowness with respect to its surrounding region. Using the lane marking start and end point information, few pixels on the lane marking are sampled. The average $H$ and $S$ values of the lane pixels are calculated. Similarly, few corresponding neighbouring pixels from the road are sampled and the average $\mathrm{H}$ and $\mathrm{S}$ of the road are computed. If the lane mark color is matching yellow hue range and the saturation range, the relative saturation with respect to background pixels is compared to confirm the color. This approach has produced reliable lane color detection under varying test scenarios. To handle the sudden intensity variations across frames, we considered color detection in ' $N$ ' frames to take the decision. The results for the absolute color method and the relative color method (proposed method) under different scenarios are shown in Table III.

Table 3 Lane color output under different scenarios

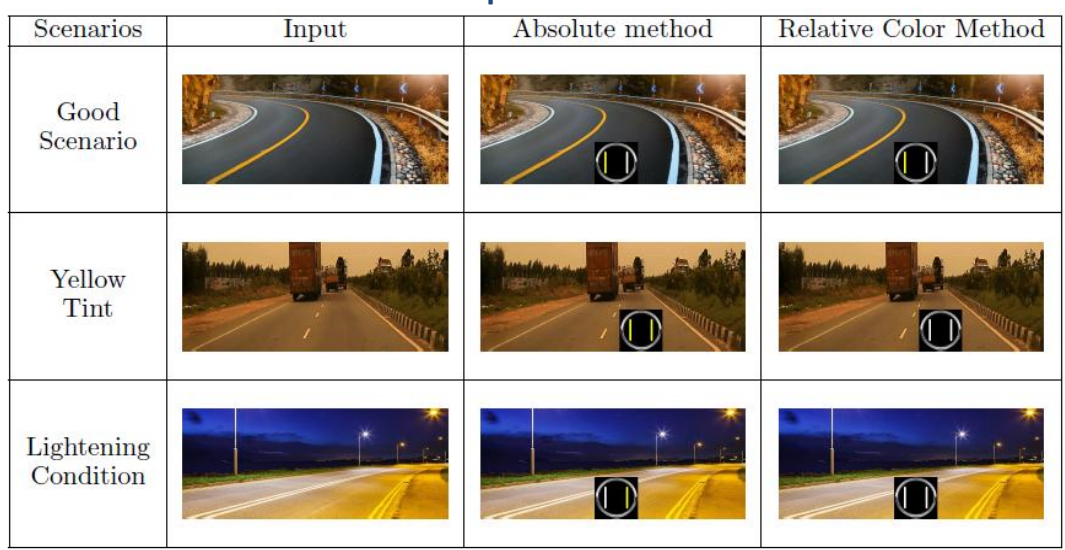

The pseudo code for the proposed method is as below.

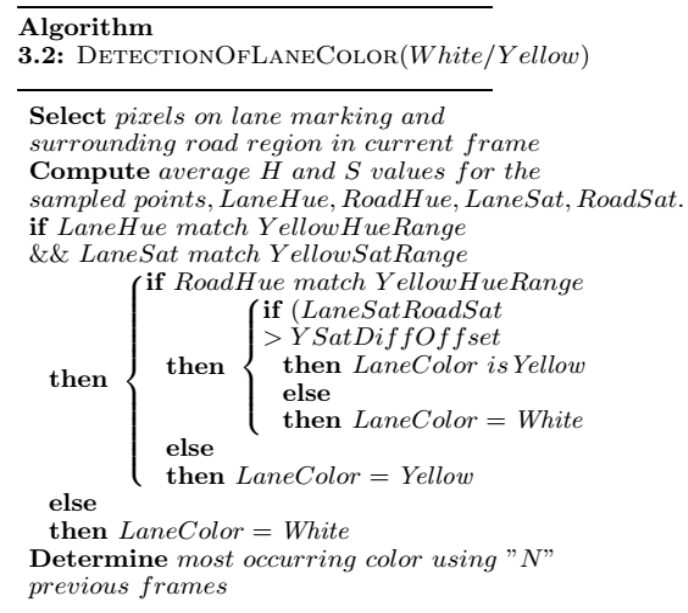

\section{Experiments and Results}

Proposed algorithm addresses the problem of identifying the type and color of lane marking under varying scenarios. According to the international standard of ISO 17361 [1] lane color and types vary between geographies. The proposed algorithm which is depicted as flow chart in Fig.7, aims to handle these variations along with other illumination variations that are faced by ADAS systems. For our experiments and validation the algorithm, we have used TCS collected data set along with standard 
Varsha Singal, Susmitha Mohan and Upendra Suddamalla; Lane Characterization Under Challenging Scenarios For Autonomous Driving System. Advances in Image and Video Processing, Volume 4 No 6, December (2016); pp: $18-26$

Caltech Dataset [13] and some challenging scenario images downloaded from internet [14]. Based on the experiments and observations, typical values are chosen for critical parameters used in proposed algorithm are shown in Table IV.

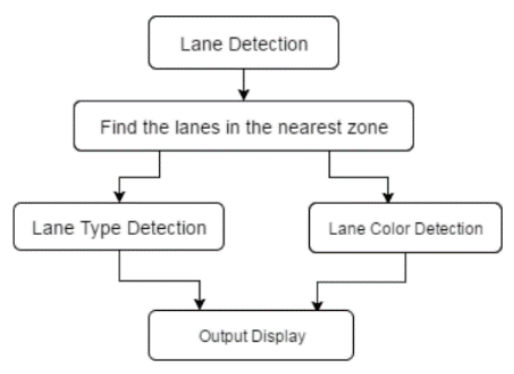

Figure 7 Flow Chart of the proposed algorithm

Table 4 Parameters and its value

\begin{tabular}{|c|c|}
\hline Parameter & Value \\
\hline $\mathrm{N}$ & 30 \\
\hline StartDistOffset & 3 \\
\hline SolidThreshold & 75 \\
\hline CountThresh & 2 \\
\hline VarThresh & 2 \\
\hline YSatDiffOffset & 30 \\
\hline
\end{tabular}

Unlike, the methods using IPM and autocorrelation which operate on single frame, the proposed method uses lane detection data across successive frames. Using the nearest zone is an advantage as the lane detection outputs are more reliable in the near zone and the classification of lane type and color is mostly required when the vehicle is at motion.

Results of the proposed algorithm for identifying the lane type and color under varying scenarios are shown in Fig.8. As depicted, our algorithm could successfully identify the lane type and color under challenging road and illumination scenarios with solid and dashed lane marks in white and yellow color.
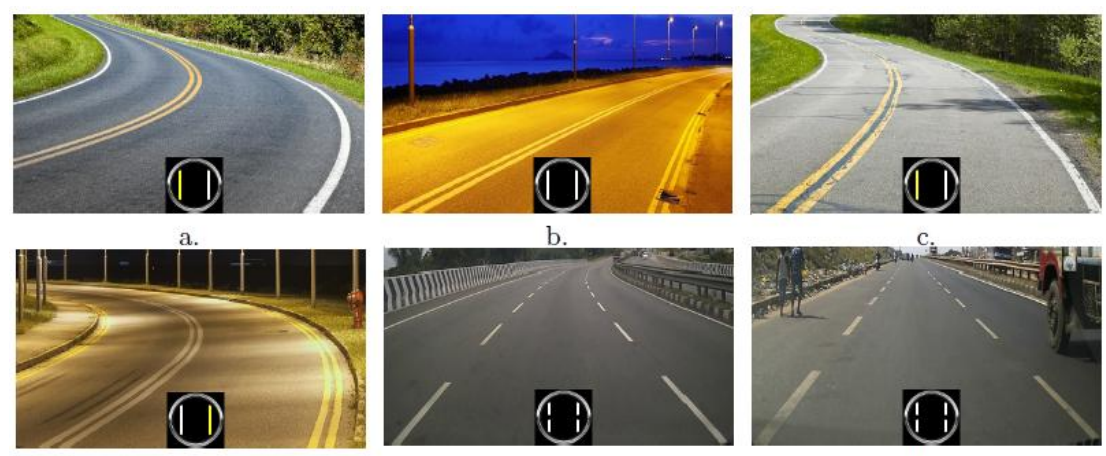

d.

e.

f.

Figure 8 Lane type and Lane color output for different scenarios

In case of fainted lane marks, where lane marking detections are not good, lane pattern is neither continuous nor periodic, so lane type is unknown shown in Fig.9, where gray lane marking represents unknown lane type. For lane color, it is assumed that lane marking detection are proper and the lane is either yellow or white in color. So, if yellow lane color is not detected, then by default lane color is white. 


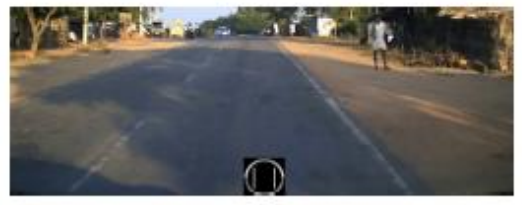

a.

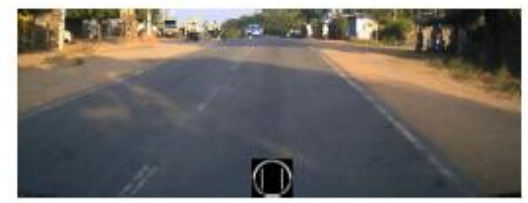

b.

Figure 9 Failure Scenarios

\section{Conclusion}

Driver assistance systems play very important role in safety of the driver and vehicle. So the features such as Lane Change Assistance, Lane Departure Warning have to be very accurate and real time. For both these functionalities, lane position, type and color are the primary information to take critical decisions. Our proposed algorithm has shown promising results on various scenarios of straight, curvy lanes with solid, dashed, white and yellow markings under good and challenging illuminations conditions. This method is capable of using the lane information from any state-of-art lane detection algorithm and performs statistical analysis with low computational power requirement. Currently we are working on enhancing this algorithm for complex weather conditions such as rain and fog.

\section{Acknowledgement}

The authors would like to thank Mr. Rajarama Nayak, Head of TCS Embedded Innovation Labs for his extensive support and valuable review comments.

\section{REFERENCES}

[1]. ISO 17361 Intelligent transport systems - Lane departure warning systems Performance requirement and test procedures, First edition 2007.

[2]. G Kaur, D Kumar: "Lane Detection Techniques: A Review," International Journal of Advanced Computer Science and Applications, vol.112, no.10, pp.0975 8887, February 2015.

[3]. U Suddamalla, S Kundu, S Farkade: “A Novel Algorithm of Lane Detection Addressing Varied Scenarios of Curved and Dashed Lanemarks," International Conference on Image Processing Theory, Tools and Applications, pp.87-92, IPTA 2015.

[4]. JM Collado, C Hilario, A De La Escalera: “Adaptative Road Lanes Detection and Classification,”

[5]. CH Rodrguez-Garavito, A Ponz, F Garca: “COMPUTER VISION APPLIED TO ROAD LINES RECOGNITION USING MACHINE LEARNING," The 7th International Conference on Information Technology, ICIT 2015.

[6]. MB de Paula, CR Jung: "Real-time detection and classification of road lane markings," 2013 XXVI Conference on Graphics, Patterns and Images, pp.1530- 1834, IEEE 2013.

[7]. S Vacek, C Schimmel, R Dillmann: “Road-marking analysis for autonomous vehicle guidance,"

[8]. S Suchitra, RK Satzoda: "Identifying Lane Types: A Modular Approach," Proceedings of the 16th International IEEE Annual Conference on Intelligent Transportation Systems, (ITSC 2013), 2013 IEEE.

[9]. LNP Boggavarapu, RS Vaddi, KR Anne: “A robust multi color lane marking detection approach for Indian scenario," International Journal of Advanced Computer Science and Applications, vol.2, no.5, 2011. 
Varsha Singal, Susmitha Mohan and Upendra Suddamalla; Lane Characterization Under Challenging Scenarios For Autonomous Driving System. Advances in Image and Video Processing, Volume 4 No 6, December (2016); pp: $18-26$

[10]. TT Tran, CS Bae, YN Kim, HM Cho, SB Cho: “An Adaptive Method for Lane Marking Detection Based on HSI Color Model," International Journal of Advanced Computer Science and Applications, ICIC 2010, CCIS 93,pp.304-311,2010.

[11]. HC Choi, SY Oh: "Illumination Invariant Lane Color Recognition by using Road Color Reference and Neural Networks," International Journal of Advanced Computer Science and Applications, 2010 IEEE.

[12]. H Lee, S Park, K Choi: "Support Vector Machines For Understanding Lane Color and Sidewalks," International Journal of Computer, Electrical, Automation, Control and Information Engineering, vol.3, no.2, 2009.

[13]. http://vision.caltech.edu/malaa/datasets/caltech-lanes/

[14]. http://www.istockphoto.com/in/stock-photos 